\title{
Transgenerational inheritance or resetting of stress-induced epigenetic modifications: two sides of the same coin
}

\author{
Penny J. Tricker* \\ Australian Centre for Plant Functional Genomics, School of Agriculture, Food and Wine, University of Adelaide, Urrbrae, SA, \\ Australia
}

\section{OPEN ACCESS}

Edited by:

Mahmoud W. Yaish,

Sultan Qaboos University, Oman

Reviewed by:

Barbara Hohn,

Friedrich Miescher Institute for Biomedical Research, Switzerland lan S. Wallace,

The University of Nevada, Reno, USA

Maja M. Klosinska,

Whitehead Institute for Biomedical

Research, USA

*Correspondence:

Penny J. Tricker,

Australian Centre for Plant Functional Genomics, School of Agriculture,

Food and Wine, University of Adelaide, Waite Campus, Hartley Grove, Urrbrae, SA 5064, Australia penny.tricker@adelaide.edu.au

Specialty section: This article was submitted to Plant Physiology,

a section of the journal

Frontiers in Plant Science

Received: 01 April 2015

Accepted: 21 August 2015

Published: 07 September 2015

Citation:

Tricker PJ (2015) Transgenerational

inheritance or resetting

of stress-induced epigenetic

modifications: two sides

of the same coin.

Front. Plant Sci. 6:699.

doi: 10.3389/fpls.2015.00699
The transgenerational inheritance of stress-induced epigenetic modifications is still controversial. Despite several examples of defense "priming" and induced genetic rearrangements, the involvement and persistence of transgenerational epigenetic modifications is not known to be general. Here l argue that non-transmission of epigenetic marks through meiosis may be regarded as an epigenetic modification in itself, and that we should understand the implications for plant evolution in the context of both selection for and selection against transgenerational epigenetic memory. Recent data suggest that both epigenetic inheritance and resetting are mechanistically directed and targeted. Stress-induced epigenetic modifications may buffer against DNA sequencebased evolution to maintain plasticity, or may form part of plasticity's adaptive potential. To date we have tended to concentrate on the question of whether and for how long epigenetic memory persists. I argue that we should now re-direct our question to investigate the differences between where it persists and where it does not, to understand the higher order evolutionary methods in play and their contribution.

Keywords: transgenerational, epigenetic, stress, re-setting, evolution, methylation, transposable elements

\section{Introduction}

Molinier et al. (2006) demonstrated that stress-induced epigenetic modification could be inherited through several generations in plants, causing considerable excitement. It had long been recognized that such capacity could allow for epigenetic priming of the progeny with important implications for improving crop plants (Mirouze and Paszkowski, 2011; Rodríguez López and Wilkinson, 2015), releasing cryptic variation (Grant-Downton and Dickinson, 2006), for population level adaptation (Richards, 2008) and adaptive evolution (Jablonka and Raz, 2009). Further examples of transgenerational epigenetic effects have been discovered including phenotypic inheritance (Verhoeven and Van Gurp, 2012) and the inheritance of gene expression (Scoville et al., 2011). Although we now have greater mechanistic understanding of transgenerational epigenetic inheritance (e.g., Crevillén et al., 2014; Kuhlmann et al., 2014) there are still few, multi-generational population or species-level studies (Richards, 2006; Bossdorf et al., 2008; Johannes et al., 2008). These few, however, have allowed us to begin to understand the evolutionary importance of stress-induced epigenetic modifications (Rapp and Wendel, 2005; Bräutigam et al., 2013; Kooke et al., 2015).

DNA cytosine methylation is an important epigenetic modification and is demonstrably heritable through mitosis. Arabidopsis epigenetic recombinant inbred line (epiRIL) populations that have no 
DNA sequence variation but epigenetic variation in DNA methylation were created by crossing wild-type "Columbia" with mutants deficient in DNA methylation. The patterns created by recombination in these Arabidopsis epiRILs led to a range of stress-tolerance and phenotypes similar to the natural range in accessions within six to nine generations (Roux et al., 2011). Highly heritable epigenetic quantitative trait loci (epiQTL) for flowering time and primary root length were found, associated with the loss of DNA methylation at differentially methylated regions in the founder line (Cortijo et al., 2014) and amenable for artificial selection. These epiRILs also displayed increased plasticity in response to drought, nutrient and salt stresses (Zhang et al., 2013; Kooke et al., 2015) and associated epiQTL were highly heritable, illustrating the stability of epigenetic modification.

\section{Stress-Induced Priming}

Biotic and abiotic stresses trigger epigenetic modifications in the genome. In particular these modifications regulate the "open-ness" of chromatin to suppress or allow gene transcription, transposition of transposable elements (TEs), nucleosome occupancy and recombination. The effect is an altered epigenome that regulates stress response. In some cases the signature of the stress experience remains in the epigenome after relief from the stress, providing a "memory" If this memory conditions the response to stress during subsequent development, the organism is said to be epigenetically primed. If the memory of the stress experienced by a parent conditions the response of its progeny, this epigenetic priming may be transgenerational. Following Molinier et al.'s (2006) demonstration of heritable epigenetic response to both biotic and abiotic stresses, transgenerational epigenetic priming of plants has been reported in response to pest and pathogen attacks (Luna et al., 2012; Rasmann et al., 2012; Slaughter et al., 2012) and to abiotic stresses including growth in high salt, UVC, heavy metal contamination, increased evaporative demand, heat and oxidative stresses (Boyko et al., 2010; Rahavi et al., 2011; Tricker et al., 2013; Matsunaga et al., 2015). Experimenters typically repeat experiments with clean seed stocks in controlled conditions and yet reproduce the same epigenetic responses, for example the priming of antibacterial defense (Dowen et al., 2012; Yu et al., 2013). These results suggest that epigenetic priming is targeted.

In Arabidopsis, priming for antibacterial defense involves active demethylation of TEs that leads to transcriptional activation of defense regulators via hormonal signals (Yu et al., 2013). DNA hyper- and hypo-methylation are observed epigenetic changes in stress response and priming (Boyko et al., 2010; Verhoeven et al., 2010; Tricker et al., 2012), and the recruitment of stress-induced methylation is regulated by small, non-coding RNAs (short-interfering RNAs/siRNAs; Boyko et al., 2010). This RNA-directed DNA methylation (RdDM) may provide DNA sequence specificity to epigenetic modifications via sequencecomplementarity of the siRNAs that recruit DNA methylation. However, DNA methylation is reversible and demethylation is also an important response and priming strategy (Yu et al., 2013; Iwasaki and Paszkowski, 2014). Molecular mechanisms that prevent the transgenerational memory of stress have been discovered (Iwasaki and Paszkowski, 2014) and these can be entrained by repeated cycles of stress (Sanchez and Paszkowski, 2014).

The challenge is to understand the dynamics of epigenetic modifications in response to stress and how these interplay with intra- or inter-generational memory to target priming.

\section{Epigenetic Inheritance of Memory}

The regulation of response to plant growth environment is clearly heritable when heritable is defined as passed from the parent to the progeny. One of the best-known examples is the regulation of vernalization requirement in winter annual Arabidopsis thaliana. The requirement for vernalizing temperatures to induce flowering is determined in the pathway involving the flowering repressor FLOWERING LOCUS C (FLC), its silencing and the epigenetic maintenance of silencing during warmer temperatures (reviewed in Baulcombe and Dean, 2014). The epigenetic regulation of vernalization in Arabidopsis is passed from parent to progeny, i.e., it is an inherited pathway. However, the accumulated epigenetic modifications themselves are reset in each sexual generation, and it is this re-setting that determines the vernalization requirement anew.

The heritable memory of epigenomic regulation is open to selection. In breeding for the epigenetic component of energy efficiency and stress tolerance in Brassica rapa (Hauben et al., 2009), the efficiency advantage of the original population and its epigenome component (phenotype, methylome, transcriptome, histone modification) was highly heritable in successive generations undergoing recurrent selection. High and low efficiency selections had distinct profiles of DNA methylation, histone methylation and acetylation different from the parent and from each other. Epigenomic profiles changed during development in opposite directions but were heritable in a cross. This did not indicate that the epigenomic profile had reached reproductive cells because it could not be fixed in the first rounds of selection. The influence of fluctuations in the environment was not explicitly investigated during this experiment, but lines bred for the epigenetic component of energy efficiency were also more drought tolerant.

\section{Re-Setting the Epigenome}

Transcriptional gene silencing is maintained by DNA methylation and histone modifications. These epigenetic modifiers are regulated during gametogenesis and are correlated with the dynamics of chromatin condensation that produce permissive and repressive states of transcriptional activation. At imprinted genes that display parent-of-origin, allele-specific expression, regulation by cytosine methylation and a Polycomb-Repressive Complex determines differential expression through cell divisions with time (reviewed in García-Aguilar and Gillmor, 2015). Methylation is re-programmed in the different nuclei during gametogenesis (Calarco et al., 2012; Jullien et al., 2012). It has been suggested that this re-programming allows the generation of mobile siRNA signals in companion cells that reinforce silencing of TEs in the embryo. In sperm and 
male germline microspore cells, asymmetric ( $\mathrm{CHH}$ sequence) methylation is reduced and 24 nucleotide siRNA from imprinted, maternally expressed genes accumulate in sperm cells. $\mathrm{CHH}$ methylation is restored after fertilization, during embryogenesis, and the pattern of DNA methylation and silencing is restored at many TEs and epialleles. However, this inheritance of silencing is progressive and incomplete in the male germline cells before fertilization (Calarco et al., 2012). Likewise, the transitions from spore mother cell to megaspore and gametophyte in the female reproductive lineage are also marked by different repressive and permissive histone composition and by chromatin remodeling, suggesting a pre-meiotic epigenetic influence on post-meiotic development (reviewed in Baroux and Autran, 2015). This re-setting during gametogenesis might allow for the removal of epigenetic modifications accumulated in response to stress or growth conditions during development of the parent. Additionally, it provides a window of opportunity to relax epigenetic suppression of transcription and transposition.

\section{The Genomic Basis of Transgenerational Epigenetic Response to Stress}

Natural variation in DNA methylation has been assayed genomewide in Arabidopsis accessions, maize and soybean inbred lines (Vaughn et al., 2007; Eichten et al., 2013; Schmitz et al., 2013a). Along with other repressive chromatin states, DNA methylation is often associated with transposon-rich centromeric regions of the genomes, recently inserted TEs or duplicated regions, and often accompanied by high concentrations of siRNAs that generate RdDM at retrotransposons (Lister et al., 2008). It has been proposed that these epigenetic mechanisms exist primarily as defenses against potentially harmful genomic elements such as TEs (reviewed in Johnson, 2007).

A number of stresses can mobilize TEs (Grandbastien, 1998) and suppression of heat stress-induced retrotransposition of the ONSEN element requires the siRNA biogenesis pathway (Ito et al., 2011). Transposons may cycle between active and silenced states and the invasion of a new TE and eventual silencing can establish epiallelism at proximal genes (MaríOrdóñez et al., 2013). Variation from new insertions may also create new, regulatory inserts responsive to the inducing stress (Ito et al., 2011) or even new, environmentally-responsive genes (reviewed in Oliver et al., 2013). Federoff (2012) has argued eloquently that, in contrast to the view that epigenetic mechanisms exist to suppress TEs, they have evolved and been preserved precisely to allow expansion, duplication and complexity derived from transposition within genomes, whilst repressing illegitimate recombination. Such a scenario requires that the suppression of TE activation by epigenetic means is relaxed or fluctuating. MaríOrdóñez et al. (2013) found that the epigenetic suppression of the newly invasive retrotransposon Evadé (EVD) was sequential so that initial, incomplete post-transcriptional silencing shifted to transcriptional silencing over generations once a copy number of 40 was reached. EVD bore the seeds of its own destruction; its molecular suppression of post-transcriptional silencing generated $\mathrm{RdDM}$ that led to its transcriptional silencing. These findings (and others reviewed in Ito and Kakutani, 2014) are consistent with the idea that TE-activation and epigenetic suppressors act in concert to allow fluctuation and complexity. It can be proposed that the re-setting of epigenetic states at gametogenesis exists to allow this relaxation.

\section{The Adaptive Potential of Transgenerational Epigenetic Responses}

Although the majority of stress-induced chromatin modifications do not persist past gametogenesis (reviewed in Pecinka and Mittelsten Scheid, 2012), others are faithfully re-acquired, albeit limited to one or a few progeny generations not exposed to the same stress (Boyko et al., 2010; Lang-Mladek et al., 2010). Pecinka and Mittelsten Scheid (2012) argued cogently that there is no conclusive evidence yet for the transgenerational epigenetic inheritance of stress-induced memory in plants, and that such evidence would need to document long-lasting changes of more than two generations that significantly influenced the plant's stress-responsiveness or adaptation. I argue that there is evidence for long-lasting epigenetically-induced change in stressresponsiveness encoded in the genome, but that it is hard to spot.

If the re-setting of stress-induced epigenetic modification at gametogenesis exists to allow encoding of transgenerational memory at new, responsive elements how might we see its signature in the genome? In some known cases, the epigenetic regulation of stress response is fixed in the genome at TEderived sequences and heritable: Examples include the siRNAbased silencing of the UBP1b gene in Arabidopsis, the AltSB aluminum tolerance locus of sorghum, and the regulation of desiccation tolerance via inducible siRNAs at the CDT-1 element of Craterostigma plantagineum (Magalhaes et al., 2007; Hilbricht et al., 2008; McCue et al., 2012). In addition, the feedback system that generated the $M u$ killer locus in maize may be highly prevalent. Via siRNAs, $M u$ killer heritably silences the $M u D R$ transposon (Slotkin et al., 2003). Mu killer derives from an inverted duplication of a partially deleted $M u D R$ element (Slotkin et al., 2005) and this derivation of the means of epigenetic silencing from the target is common in many genomes (reviewed in Lisch, 2013) and is subject to purifying selection, at least in rice (Hanada et al., 2009). Coupled with evidence that TE insertions increase the number of stress-responsive genes (Naito et al., 2009) these reports suggest that the relaxation of epigenetic suppression of TEs forms part of an evolvable genomic memory, but that this is largely invisible over evolutionary timescales (Lisch, 2013).

Reversible epigenetic regulation may have advantages in fluctuating environments. Burggren (2015) suggested that the immediate "sunsetting" of a stress-induced epigenetic modification once the stress was removed, could allow for bethedging against the possible return of the stress. The progeny of one generation exposed to a stress would maintain the epigenetic capacity to respond but not the stressed phenotype. Being poised for fluctuation-that is having an extra layer of regulation ready for release-would benefit survival if the stress was encountered again. Alternatively, epigenetic phenotypic modifications might "wash-out" over generations so that the phenotypic effects would 


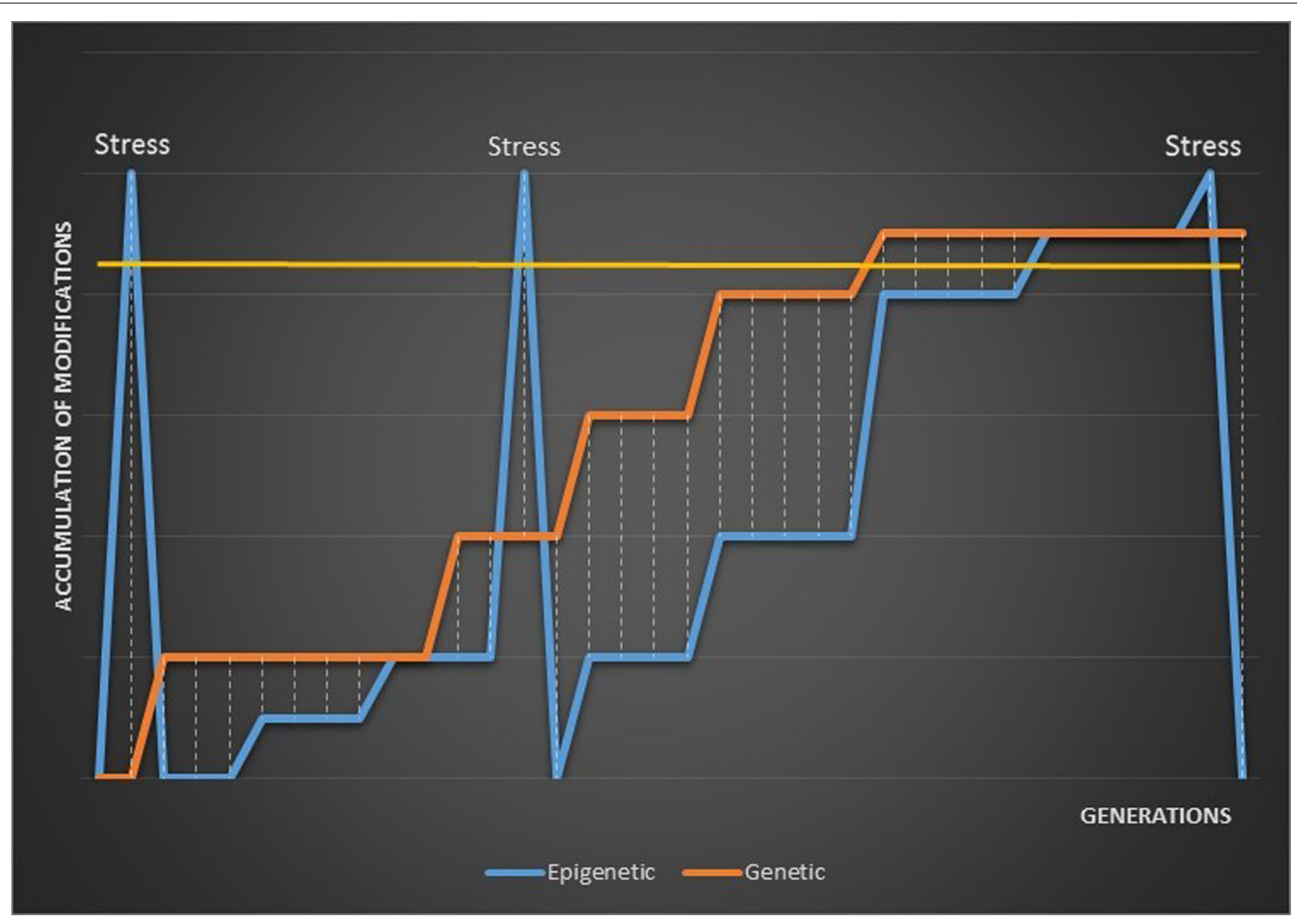

FIGURE 1 | A model of the distance between epigenetic and genetic variation when epigenetic modifications accumulate following stress and are re-set between generations. Phenotypic variation is only visible above the gold, horizontal line. The distance between epigenetic modifications and accumulating genetic mutations is illustrated by the dashed lines.

become undetectable or disappear at population scale (Burggren, 2015).

Epigenetic and genetic variation co-evolve (Schmitz et al., $2013 b)$. This needs to happen so that epigenetic plasticity does not completely buffer evolvability and reduce the correlation between fitness and genotype, slowing selection. Klironomos et al. (2013) modeled the effect of selection on epigenetic as well as genetic variation. They showed that early selection of epigenetic variation could allow for the build-up of neutral genetic variation and faster adaptation in comparison with selection via genetic variation alone. This would allow for population survival by epigenetic adaptation following stress, and act as a stepping-stone to increased genetic fitness. Once genetic fitness had increased and been fixed, epigenetic variations would accumulate neutrally.

These models might account for the seeming variability in observations of epigenetic phenotypic plasticity and transgenerational epigenetic responses to stress. A rapid accumulation of epigenetic variation in response to stress would be visible in the phenotype of the first one or several generations if washed-out or rapidly sunset. Alternatively, a stress-induced burst of epigenetic modification might be visible in the first generation but largely disappear after re-setting with only a proportion fixed following the re-set generation. A rapid resetting of stress-induced epigenetic variation followed by neutral accumulation to a new fitness maximum would only become visible once epigenetic variation exceeded genetic variation, or following another stress-induced burst. This model is illustrated in Figure 1.

Theoretically a brief, transgenerational epigenetic memory ensures plasticity, but the dual inheritance of genetic and epigenetic variation ensures adaptation (Pal, 1998). If re-setting allows a window of opportunity for increased epigenetic variation in response to stress, then it could form a vital part of a species' evolvability. Importantly, mechanistic investigation of re-setting over only a few generations should elucidate the contribution of re-setting to adaptation where, over longer timescales, the evidence of new stress-responsiveness from the activation or exaption of TEs will be invisible. Likewise the recent discovery of molecular mechanisms that restrict transgenerational epigenetic inheritance (Iwasaki and Paszkowski, 2014) will contribute to our understanding of the targeting of epigenetic re-setting or inheritance.

\section{Conclusion}

When we consider the evidence for epigenetic transgenerational inheritance in response to stress we should consider whether it will be visible in an individual epigenome and whether the ebb and flow of visible epigenetic modifications limits or contributes to plasticity. The framework for population and species-level studies 
of genetics can now be applied in epigenetics to inform our understanding (Richards, 2006; Johnson and Tricker, 2010). It is to be hoped that the combination of mechanistic and theoretical understanding advancing side by side, and the recognition that the persistence or reversibility of transgenerational epigenetic modifications are really two sides of the same coin, will allow us to exploit the undoubted potential of epigenetic regulation of plant stress response for the future.

\section{References}

Baroux, C., and Autran, D. (2015). Chromatin dynamics during cellular differentiation in the female reproductive lineage of flowering plants. Plant $\mathrm{J}$. 83, 160-176. doi: 10.1111/tpj.12890

Baulcombe, D. C., and Dean, C. (2014). Epigenetic regulation in plant responses to the environment. Cold Spring Harb. Perspect. Biol. 6:a019471. doi: 10.1101/cshperspect.a019471

Bossdorf, O., Richards, C. L., and Pigliucci, M. (2008). Epigenetics for ecologists. Ecol. Lett. 11, 106-115. doi: 10.1111/j.1461-0248.2007.01130.x

Boyko, A., Blevins, T., Yao, Y., Golubov, A., Bilichak, A., Ilnytskyy, Y., et al. (2010). Transgenerational adaptation of Arabidopsis to stress requires DNA methylation and the function of Dicer-like proteins. PLoS ONE 5:e9514. doi: 10.1371/journal.pone.0009514

Bräutigam, K., Vining, K. J., Lafon-Placette, C., Fossdal, C. G., Mirouze, M., Guitierrez Marcos, J., et al. (2013). Epigenetic regulation of adaptive responses of forest tree species to the environment. Ecol. Evol. 3, 399-415. doi: $10.1002 /$ ece 3.461

Burggren, W. W. (2015). Dynamics of epigenetic phenomena: intergenerational and intragenerational phenotype 'washout'. J. Exp. Biol. 218, 80-87. doi: 10.1242/jeb. 107318

Calarco, J. P., Borges, F., Donoghue, M. T. A., Van Ex, F., Jullien, P. E., Lopes, T., et al. (2012). Reprogramming of DNA methylation in pollen guides epigenetic inheritance via small RNA. Cell 151, 194-205. doi: 10.1016/j.cell.2012.09.001

Cortijo, S., Wardenaar, R., Colomé-Tatché, M., Gilly, A., Etcheverry, M., Labadie, K., et al. (2014). Mapping the epigenetic basis of complex traits. Science 343, 1145-1148. doi: 10.1126/science.1248127

Crevillén, P., Yang, H., Cui, X., Greeff, C., Trick, M., Qiu, Q., et al. (2014). Epigenetic reprogramming that prevents transgenerational inheritance of the vernalized state. Nature 515, 587-590. doi: 10.1038/nature13722

Dowen, R. H., Pelizzola, M., Schmitz, R. J., Lister, R., Dowen, J. M., Nery, J. R., et al. (2012). Widespread dynamic DNA methylation in response to biotic stress. Proc. Natl. Acad. Sci. U.S.A. 109, E2183-E2191. doi: 10.1073/pnas.1209329109

Eichten, S. R., Briskine, R., Song, J., Li, Q., Swanson-Wagner, R., Hermanson, P. J., et al. (2013). Epigenetic and genetic influences on DNA methylation variation in maize populations. Plant Cell 25, 2783-2797. doi: 10.1105/tpc.113.114793

Federoff, N. V. (2012). Transposable elements, epigenetics, and genome evolution. Science 338, 758-767. doi: 10.1126/science.338.6108.758

García-Aguilar, M., and Gillmor, C. S. (2015). Zygotic genome activation and imprinting: parent-of-origin gene regulation in plant embryogenesis. Curr. Opin. Plant Biol. 27, 29-35. doi: 10.1016/j.pbi.2015.05.020

Grandbastien, M.-A. (1998). Activation of plant retrotransposons under stress conditions. Trends Plant Sci. 3, 181-187.

Grant-Downton, R. T., and Dickinson, H. G. (2006). Epigenetics and its implications for plant biology 2. The 'epigenetic epiphany': epigenetics, evolution and beyond. Ann. Bot. 97, 11-27. doi: 10.1093/aob/mcj001

Hanada, K., Vallejo, V., Nobuta, K., Slotkin, R. K., Lisch, D., Meyers, B. C., et al. (2009). The functional role of pack-MULEs in rice inferred from purifying selection and expression profile. Plant Cell 21, 25-38. doi: $10.1105 /$ tpc. 108.063206

Hauben, M., Haesendonckx, B., Standaert, E., Van Der Kelen, K., Azmi, A., Akpo, H., et al. (2009). Energy use efficiency is characterized by an epigenetic component that can be directed through artificial selection to increase yield. Proc. Natl. Acad. Sci. U.S.A. 106, 20109-20114. doi: 10.1073/pnas.0908755106

Hilbricht, T., Varotto, S., Sgaramella, V., Bartels, D., Salamini, F., and Furini, A. (2008). Retrotransposons and siRNA have a role in the evolution of desiccation tolerance leading to resurrection of the plant Craterostigma plantagineum. New Phytol. 179, 877-887. doi: 10.1111/j.1469-8137.2008.02480.x

\section{Acknowledgments}

I thank Carlos Rodriguez Lopez and three anonymous reviewers for critical comments on earlier drafts of the manuscript. PT's work is supported by the Premier's Research and Industry Fund grant provided by the South Australian Government Department of State Development (IRGP15) and the Australian Centre for Plant Functional Genomics.

Ito, H., Gaubert, H., Bucher, E., Mirouze, M., Vaillant, I., and Paszkowski, J. (2011). An siRNA pathway prevents transgenerational retrotransposition in plants subjected to stress. Nature 472, 115-119. doi: 10.1038/nature09861

Ito, H., and Kakutani, T. (2014). Control of transposable elements in Arabidopsis thaliana. Chromosome Res. 22, 217-223. doi: 10.1007/s10577-014-9417-9

Iwasaki, M., and Paszkowski, J. (2014). Identification of genes preventing transgenerational transmission of stress-induced epigenetic states. Proc. Natl. Acad. Sci. U.S.A. 111, 8547-8552. doi: 10.1073/pnas.1402275111

Jablonka, E., and Raz, G. (2009). Transgenerational epigenetic inheritance: prevalence, mechanisms, and implications for the study of heredity and evolution. Q. Rev. Biol. 84, 131-176. doi: 10.1086/598822

Johannes, F., Colot, V., and Jansen, R. C. (2008). Epigenome dynamics: a quantitative genetics perspective. Nat. Rev. Genet. 9, 883-890. doi: $10.1038 / \operatorname{nrg} 2467$

Johnson, L. J. (2007). The genome strikes back: the evolutionary importance of defence against mobile elements. Evol. Biol. 34, 121-129. doi: 10.1007/s11692007-9012-5

Johnson, L. J., and Tricker, P. J. (2010). Epigenomic plasticity within populations: its evolutionary significance and potential. Heredity 105, 113-121. doi: 10.1038/hdy.2010.25

Jullien, P. E., Susaki, D., Yelagandula, R., Higashiyama, T., and Berger, F. (2012). DNA methylation dynamics during sexual reproduction in Arabidopsis thaliana. Curr. Biol. 22, 1825-1830. doi: 10.1016/j.cub.2012.07.061

Klironomos, F. D., Berg, J., and Collins, S. (2013). How epigenetic mutations can affect genetic evolution: model and mechanism. Bioessays 35, 571-578. doi: 10.1002/bies.201200169

Kooke, R., Johannes, F., Wardenaar, R., Becker, F., Etcheverry, M., Colot, V., et al. (2015). Epigenetic basis of morphological variation and phenotypic plasticity in Arabidopsis thaliana. Plant Cell 27, 337-348. doi: 10.1105/tpc.114.133025

Kuhlmann, M., Finke, A., Mascher, M., and Mette, M. F. (2014). DNA methylation maintenance consolidates RNA-directed DNA methylation and transcriptional gene silencing over generations in Arabidopsis thaliana. Plant J. 80, 269-281. doi: $10.1111 /$ tpj. 12630

Lang-Mladek, C., Popova, O., Kiok, K., Berlinger, M., Rakic, B., Aufsatz, W., et al. (2010). Transgenerational inheritance and resetting of stress-induced loss of epigenetic gene silencing in Arabidopsis. Mol. Plant 3, 594-602. doi: $10.1093 / \mathrm{mp} / \mathrm{ssq} 014$

Lisch, D. (2013). How important are transposons for plant evolution? Nat. Rev. Genet. 14, 49-61. doi: 10.1038/nrg3374

Lister, R., O’Malley, R. C., Tonti-Filippini, J., Gregory, B. D., Berry, C. C., Millar, A. H., et al. (2008). Highly integrated single-base resolution maps of the epigenome in Arabidopsis. Cell 133, 523-536. doi: 10.1016/j.cell.2008.03.029

Luna, E., Bruce, T. J. A., Roberts, M. R., Flors, V., and Ton, J. (2012). Nextgeneration systemic acquired resistance. Plant Physiol. 158, 844-853. doi: $10.1104 /$ pp.111.187468

Marí-Ordóñez, A., Marchais, A., Etcheverry, M., Martin, A., Colot, V., and Voinnet, O. (2013). Reconstructing de novo silencing of an active plant retrotransposon. Nat. Genet. 45, 1029-1039. doi: 10.1038/ng.2703

Magalhaes, J. V., Liu, J., Guimarăes, C. T., Lana, U. G. P., Alves, V. M. C., Wang, Y.H., et al. (2007). A gene in the multidrug and toxic compound extrusion (MATE) family confers aluminum tolerance in sorghum. Nat. Genet. 39, 1156-1161. doi: $10.1038 /$ ng2074

Matsunaga, W., Ohama, N., Tanabe, N., Masuta, M., Masuda, S., Mitani, N., et al. (2015). A small RNA mediated regulation of a stress-activated retrotransposon and the tissue specific transposition during the reproductive period in Arabidopsis. Front. Plant Sci. 6:48. doi: 10.3389/fpls.2015.00048

McCue, A. D., Nuthikattu, S., Reeder, S. H., and Slotkin, R. K. (2012). Gene expression and stress response mediated by the epigenetic regulation 
of a transposable element small RNA. PLoS Genet. 8:e1002474. doi: 10.1371/journal.pgen.1002474

Mirouze, M., and Paszkowski, J. (2011). Epigenetic contribution to stress adaptation in plants. Curr. Opin. Plant Biol. 14, 267-274. doi: 10.1016/j.pbi.2011.03.004

Molinier, J., Ries, G., Zipfel, C., and Hohn, B. (2006). Transgeneration memory of stress in plants. Nature 442, 1046-1049. doi: 10.1038/nature05022

Naito K., Zhang, F., Tsukiyama, T., Saito, H., and Hancok, C.N. (2009). Unexpected consequences of a sudden and massive transposon amplification on rice gene expression. Nature 461, 1130-1134. doi: 10.1038/nature08479

Oliver, K. R., McComb, J. A., and Greene, W. K. (2013). Transposable elements: Powerful contributors to angiosperm evolution and diversity. Genome Biol. Evol. 5, 1886-1901. doi: 10.1093/gbe/evt141

Pal, C. (1998). Plasticity, memory and the adaptive landscape of the genotype. Proc. R. Soc. Lond. B 265, 1319-1323. doi: 10.1098/rspb.1998.0436

Pecinka, A., and Mittelsten Scheid, O. (2012). Stress-induced chromatin changes: a critical view on their heritability. Plant Cell Physiol. 53, 801-808. doi: $10.1093 / \mathrm{pcp} / \mathrm{pcs} 044$

Rahavi, M. R., Migicovsky, Z., Titov, V., and Kovalchuk, I. (2011). Transgenerational adaptation to heavy metal salts in Arabidopsis. Front. Plant Sci. 2:91. doi: $10.3389 /$ fpls.2011.00091

Rapp, R. A., and Wendel, J. F. (2005). Epigenetics and plant evolution. New Phytol. 168, 81-91. doi: 10.1111/j.1469-8137.2005.01491.x

Rasmann, S., De Vos, M., Casteel, C. L., Tian, D., Halitschke, R., Sun, J. Y., et al. (2012). Herbivory in the previous generation primes plants for enhanced insect resistance. Plant Physiol. 158, 854-863. doi: 10.1104/pp.111.187831

Richards, E. J. (2006). Inherited epigenetic variation-revisiting soft inheritance. Nat. Rev. Genet. 7, 395-401. doi: 10.1038/nrg1834

Richards, E. J. (2008). Population epigenetics. Curr. Opin. Genet. Dev. 18, 221-226. doi: 10.1016/j.gde.2008.01.014

Rodríguez López, C. M., and Wilkinson, M. J. (2015). Epi-fingerprinting and epiinterventions for improved crop production and food quality. Front. Plant Sci. 6:397. doi: $10.3389 /$ fpls.2015.00397

Roux, F., Tatché, M. C., Edelist, C., Wardenaar, R., Guerche, P., Hospital, F., et al. (2011). Genome-wide epigenetic perturbation jump starts patterns of heritable variation found in nature. Genetics 188, 1015-1017. doi: 10.1534/genetics.111.128744

Sanchez, D. H., and Paszkowski, J. (2014). Heat-induced release of epigenetic silencing reveals the concealed role of an imprinted plant gene. PLoS Genet. 10:11. doi: 10.1371/journal.pgen.1004806

Schmitz, R. J., He, Y., Valdés-López, O., Khan, S. M., Joshi, T., Urich, M. A., et al. (2013a). Epigenome-wide inheritance of cytosine methylation variants in a recombinant inbred population. Genome Res. 23, 1663-1674. doi: $10.1101 /$ gr. 152538.112

Schmitz, R. J., Schultz, M. D., Urich, M. A., Nery, J. R., Pelizzola, M., Libiger, O., et al. (2013b). Patterns of population epigenomic diversity. Nature 495, 193-200. doi: $10.1038 /$ nature 11968

Scoville, A. G., Barnett, L. L., Bodbyl-Roels, S., Kelly, J. K., and Hileman, L. C. (2011). Differential regulation of a MYB transcription factor is correlated with transgenerational epigenetic inheritance of trichome density in Mimulus guttatus. New Phytol. 191, 251-263. doi: 10.1111/j.1469-8137.2011. 03656.x

Slaughter, A., Daniel, X., Flors, V., Luna, E., Hohn, B., and Mauch-Mani, B. (2012). Descendants of primed Arabidopsis plants exhibit resistance to biotic stress. Plant Physiol. 158, 835-843. doi: 10.1104/pp.111. 191593

Slotkin, R. K., Freeling, M., and Lisch, D. (2003). Mu killer causes the heritable inactivation of the Mutator family of transposable elements in Zea mays. Genetics 165, 781-797.

Slotkin, R. K., Freeling, M., and Lisch, D. (2005). Heritable transposon silencing initiated by a naturally occurring transposon inverted duplication. Nat. Genet. 37, 641-644. doi: 10.1038/ng1576

Tricker, P. J., Gibbings, J. G., Rodríguez López, C. M., Hadley, P., and Wilkinson, M. J. (2012). Low relative humidity triggers RNA-directed de novo DNA methylation and suppression of genes controlling stomatal development. J. Exp. Bot. 63, 3799-3813. doi: 10.1093/jxb/ers076

Tricker, P. J., Rodríguez López, C. M., Hadley, P., Wagstaff, C., and Wilkinson, M. J. (2013). Pre-conditioning the epigenetic response to high vapor pressure deficit increases the drought tolerance of Arabidopsis thaliana. Plant Signal. Behav. 8, e25974. doi: $10.4161 / \mathrm{psb} .25974$

Vaughn, M. W., Tanurdžić, M., Lippman, Z., Jiang, H., Carrasquillo, R., Rabinowicz, P. D., et al. (2007). Epigenetic natural variation in Arabidopsis thaliana. PLoS Biol. 5:e174. doi: 10.1371/journal.pbio.0050174

Verhoeven, K. J. F., and Van Gurp, T. P. (2012). Transgenerational effects of stress exposure on offspring phenotypes in apomictic dandelion. PLoS ONE 7:e38605. doi: 10.1371/journal.pone.0038605

Verhoeven, K.J.F., Jansen, J.J., van Dijk, P.J., Biere, A. (2010). Stress-induced DNA methylation changes and their heritability in asexual deandelions. New Phytol. 185, 1108-1118. doi: 10.1111/j.1469-8137.2009.03121.x

Yu, A., Lepèrea, G., Jay, F., Wang, J., Bapaume, L., Wang, Y., et al. (2013). Dynamics and biological relevance of DNA demethylation in Arabidopsis antibacterial defense. Proc. Natl. Acad. Sci. U.S.A. 110, 2389-2394. doi: 10.1073/pnas.1211757110

Zhang, Y.-Y., Fischer, M., Colot, V., and Bossdorf, O. (2013). Epigenetic variation creates potential for evolution of plant phenotypic plasticity. New Phytol. 197, 314-322. doi: $10.1111 /$ nph. 12010

Conflict of Interest Statement: The author declares that the research was conducted in the absence of any commercial or financial relationships that could be construed as a potential conflict of interest.

Copyright $\odot 2015$ Tricker. This is an open-access article distributed under the terms of the Creative Commons Attribution License (CC BY). The use, distribution or reproduction in other forums is permitted, provided the original author(s) or licensor are credited and that the original publication in this journal is cited, in accordance with accepted academic practice. No use, distribution or reproduction is permitted which does not comply with these terms. 